\title{
On Some Nonstandard Asymptotic Approximation of Integrals and
} Series

Tahir H. Ismail

Ibrahim O. Hamad Rashad R. Haji

tahir_hsis@uomosul.edu.iq ibrahim.hamad@su.edu.krdrashad.haji@su.edu.krd College of Computer Sciences

College of Sciences

and Mathematics, University of Mosul

University of Salahaddin

Received on: 04/09/2004

Accepted on: 05/04/2005

\section{ABSTRACT}

The aim of this paper is to use some concepts of nonstandard analysis given by Robinson, A. and axiomataized by Nelson, E. to prove some theorems concerning the approximation of integrals and the convergence of sequences and series.

Keywords: nonstandard asymptotic, integral, series.

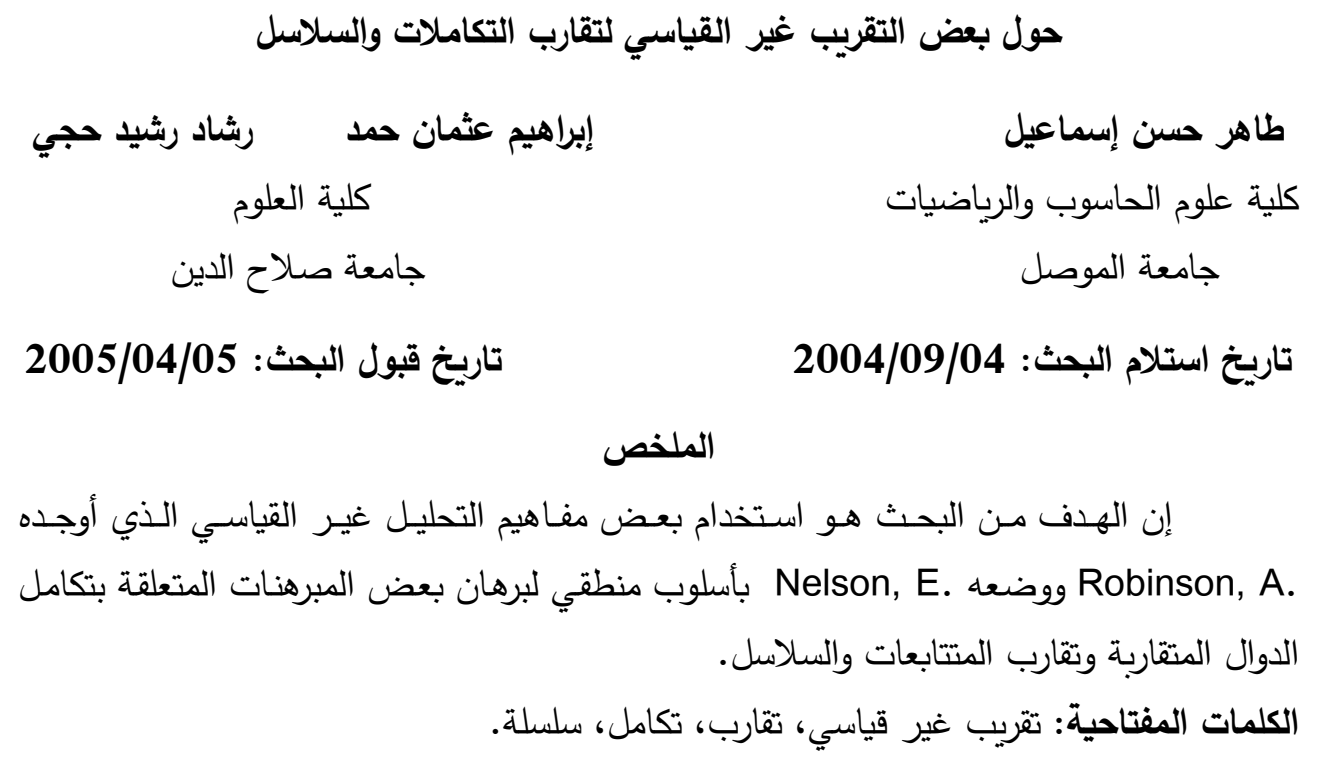

\section{INTRODUCTION:}

Through this paper we consider properties of a quite general nature, which unify certain number of processes used to establish approximate expression of numbers and functions.

Finally, we shall explain our nonstandard works and we treat the following problems.

a) How to approximate certain functions to other functions?

b) How to calculate the proper and improper integrals for approximate functions? 
c) What is the method to transform an unlimited integrand to another, everywhere limited?

d) How to find an appreciable length for functions which are infinitesimal?

e) How to calculate the sum of integral sequences under certain nonstandard conditions?

Throughout this paper the following definitions and notations will be used:

A real number $\mathrm{x}$ is called infinitesimal if and only if $|x|<r$ for all $\mathrm{r} \in \mathrm{R}$.

If $x$ and $y$ are real numbers, then $x$ and $y$ are said to be infinitely near, (denoted by $x \cong y$ ), if $x-y$ is infinitesimal.

A real number $x$ is called limited if $|x| \leq r$ for some $r \in \mathrm{R}^{+}$.

A real number $x$ is called appreciable (denoted by $A^{+}$), if $x$ is limited but not infinitesimal.

A real number $x$ is called unlimited if $|x|>r$ for all $r \in \mathrm{R}^{+}$.

The collection of limited, unlimited real numbers of infinitesimals are called external sets [3], [5].

The external set of infinitesimal real numbers is called the monad of 0 (denoted by $\mathrm{m}(0)$ ). In general, the set of all real numbers, which are infinitely near to a standard real number $a$, is called the monad of $a$, (denoted by $\mathrm{m}(\mathrm{a}))$ [6], [7].

The set of all limited real numbers is called principal galaxy, (denoted by G).

For any real number $a$, the set of all real numbers $x$ such that $x-a$ limited is called the galaxy of $a$ (denoted by $\mathrm{G}(\mathrm{a})$ ) [2].

Let $\alpha(\alpha \neq 0)$ and $x \in R$,we define the $\alpha$-galaxy(x) as follows: $\alpha-\operatorname{galaxy}(x)=\left\{y \in R: \frac{y-x}{\alpha}\right.$ is limited $\}$ and denoted by $\alpha-G(x)$ [2].

\section{THE MAIN RESULTS:}

\section{Asymptotic Approximation of Integrals}

Theorem (I,1): If $f$ and $g$ are two measurable internal functions such that $f(x) \cong g(x)$ for all $x \in[a, b]$ of limited length, then $\int_{a}^{b} f(x) \cong \int_{a}^{b} g(x)$ 
Proof: Define the function $h:[a, b] \rightarrow R$ by $h(x)=f(x)-g(x)$ then $h(x) \cong 0$ for every $x \in[a, b]$. Let $S=\sup _{x \in[a, b]}|h(x)|$. Then $S \cong 0$. Hence $\left|\int_{a}^{b} f(x) d x-\int_{a}^{b} g(x) d x\right| \leq \int_{a}^{b}|h(x) d x| \leq S(b-a) \cong 0$ therefore $\int_{a}^{b} f(x) \cong \int_{a}^{b} g(x)$

In the following theorem, we form a condition in order that the approximate equality of Theorem (I.1) holds for unbounded intervals.

Theorem(I,2): Let $f$ and $g$ be two measurable internal functions such that $f(x) \cong g(x)$ for every limited $x$. Let $h$ be an integrable standard function such that $|f(x)|,|g(x)| \leq h(x)$ for every $x \in R$. Then $\int_{-\infty}^{\infty} f(x) d x \cong \int_{-\infty}^{\infty} g(x) d x$

Proof: By Theorem (I.1) for every limited $n \in N$ we have

$$
\int_{-n}^{n} f(x) d x-\int_{-n}^{n} g(x) d x
$$

By Robinson Lemma [2] there exist a non-limited $\gamma \in N$ such that

$$
\int_{-\gamma}^{\gamma} f(x) d x-\int_{-\gamma}^{\gamma} g(x) d x
$$

Since $h(x)$ is integrable standard function, we have $\int_{|x|>\gamma} h(x) d x \cong 0$.

Hence $\quad \int_{|x|>\gamma} f(x) d x \cong 0$, and $\int_{|x|>\gamma} g(x) d x \cong 0$.

Therefore $\int_{-\gamma}^{\gamma} f(x) d x=\int_{-\infty}^{\infty} f(x) d x$ and $\int_{-\gamma}^{\gamma} g(x) d x=\int_{-\infty}^{\infty} g(x) d x$.

We conclude that $\int_{-\infty}^{\infty} f(x) d x \cong \int_{-\infty}^{\infty} g(x) d x$

The above properties (Theorem (I,1) and (I,2)) permit, particularly to give an approximate value to the integral of a function which is near a function with known primitive. Notice that in the proof of Theorem (I.2), the major function $h$ interferes only in the justification, and did not interfere in the approximation's quality. 
Theorem (I, 3): Let $f$ and $g$ be two measurable internal functions such that $f(x) \cong g(x)$ for $x \in A^{+}$(the set of appreciable numbers). Let $h$ be an integrable standard function such that $|f(x)|,|g(x)| \leq h(x)$ for every $x \in R^{+}$.

Then $\int_{0}^{\infty} f(x) d x \cong \int_{0}^{\infty} g(x) d x$

Example (I.4): Let $t>0$ and $p$ be standard, and $\omega$ be unlimited positive integer, define $f(v)$ and $J(t)$ as follows:

$$
f(v)=\left\{\begin{array}{ccc}
\frac{(t-v)^{\omega-1}}{(1+v)^{\omega+p}} & \text { if } & 0 \leq v \leq t \\
0 & \text { if } & v>t
\end{array} \text { and } J(t)=\int_{0}^{t} f(v)\right.
$$

The maximum of the integrand attains for $v=0$, putting this maximum as a factor, we obtain

$$
J(t)=t^{\omega-1} \int_{0}^{t} \frac{\left(1-\frac{v}{t}\right)^{\omega-1}}{(1+v)^{\omega+p}} d v=t^{\omega-1} \int_{0}^{t} g(v) d v
$$

The new integrand is limited everywhere. By using the Euler formula $\left(1-\frac{y}{\omega}\right)^{\omega} \cong e^{-y}$ we see that the passage from the appreciable to the infinitesimal for the integrand effected when leaving the $\frac{t}{\omega}-$ galaxy. We obtain an approximately standard integrand, by $\operatorname{setting} v=\frac{t u}{\omega}$. Then $J=\frac{t^{\omega}}{\omega} \int_{0}^{\omega} \frac{\left(1-\frac{u}{\omega}\right)^{\omega-1}}{\left(1+\frac{t u}{\omega}\right)^{\omega+p}} d u$

Let $I(u)=\left\{\begin{array}{llr}\frac{\left(1-\frac{u}{\omega}\right)^{\omega-1}}{\left(1+\frac{t u}{\omega}\right)^{\omega+p}} & \text { if } & 0 \leq u \leq \omega \\ 0 & \text { if } & u>\omega\end{array}\right.$.

Then for every limited $u \geq 0, I(u)=e^{(1+t) u}$ and for every $u \geq 0$, $I(u) \leq\left(1-\frac{u}{\omega}\right)^{\omega-1}=e^{(\omega-1) \log \left(1-\frac{u}{\omega}\right)} \leq e^{\left(\frac{\omega-1}{\omega}\right) u} \leq e^{-\frac{u}{2}}$

Hence, by Theorem (I.2) $\int_{0}^{\infty} I(u) d u \cong \int_{0}^{\infty} e^{-(1+t) u} d u=\frac{1}{1+t}$ 
Consequently $J(t)=(1+a) \frac{t^{\omega}}{\omega(1+t)},(a \cong 0)$

Comments on the strategy which comes out from example (I.4):

The above example is an illustration of the situation for which Theorem (I.2) is applied particularly, and of the procedure to follow. First of all we observe that Theorem (I.2) is mainly concerned with the functions $f$, which are: i) Limited every where, ii) noninfinitesimals on a subset of $R$, which contains at least one interval of appreciable length, and does not exceed the principal galaxy[2]. In fact, if $\{x: f(x) \cong 0\} \supseteq G$, we can not find a standard majoration of $f$, and for example if $\{x: f(x) \cong 0\} \subset m(0), \quad$ we get $\quad \int_{0}^{\infty} f(x) d x \cong 0$ which implies that $\int_{0}^{\infty} g(x) d x \cong 0$. Now such approximation of an infinitesimal number by another infinitesimal number is not meaningful

In order to be able to treat the case of an integrand, which does not satisfy the conditions (i) and (ii), we examine the reason in the Example (I.4). The departure function $f(v)$ is not necessarily limited everywhere, and $\{v: f(v) \cong 0\} \subset m(0)$.

1) We obtain the integrand $g(v)$ which is limited everywhere by putting maximum of $f(v)$ in factor.

2) We know that $\{v: f(v)=0\}$ is equal to the positive part of $\frac{t}{\omega}-$ galaxy

This observation guides us to the choice of the variable changement $v=\frac{t u}{\omega}$, after which we obtain the integrand $I(u)$ which satisfies the conditions (i) and (ii).

\section{Asymptotic Approximation of Series}

The following theorem links the series and integrals.

Theorem (II.1): Let $f$ be a positive, decreasing function such that $f(0) \cong 0$ then for every $k \in N$

i) $\sum_{n=0}^{k} f(n) \cong \int_{0}^{k} f(x) d x$ 
ii) $\sum_{n=0}^{\infty} f(n)$ is convergent if and only if $\int_{0}^{\infty} f(x) d x$ is convergent .In the case of convergence we have $\sum_{n=0}^{\infty} f(n) \cong \int_{0}^{\infty} f(x) d x$.

\section{Proof:}

i) Since $\sum_{n=0}^{k} f(n) \geq \int_{0}^{k} f(x) d x \geq \sum_{n=1}^{k+1} f(n)$. Therefore $\sum_{n=0}^{k} f(n)-\sum_{n=1}^{k+1} f(n)=f(0)-f(k+1) \cong 0$. Hence $\quad \sum_{n=0}^{k} f(n) \cong \int_{0}^{k} f(x) d x$.

ii) Since $\sum_{n=0}^{\infty} f(n) \geq \int_{0}^{\infty} f(x) d x \geq \sum_{n=1}^{\infty} f(n), \sum_{n=0}^{\infty} f(n)$ is convergent if and only if $\int_{0}^{\infty} f(x) d x$ is convergent. In the case of convergence, we have $\sum_{n=0}^{\infty} f(n) \cong \int_{0}^{\infty} f(x) d x$

Finally we give some asymptotic theorems on the series. Theorem (II.2) is a version of Theorem (I.3).

Theorem (II.2): Let $\left\{u_{n}\right\}_{n \in \mathbf{N}}$ and $\left\{v_{n}\right\}_{n \in \mathbf{N}}$ be two internal sequences such that $u_{n} \cong v_{n}$ for every $n \in N$. Let $\sum_{n=0}^{\infty} w_{n}$ be a convergent standard series such that $\left|u_{n}\right|,\left|v_{n}\right| \leq w_{n}$ for every $\mathrm{n} \in \mathbf{N}$, then $\sum_{n=0}^{\infty} u_{n} \cong \sum_{n=0}^{\infty} v_{n}$.

The following theorems are special cases for the above theorem; they facilitate the calculations of the theoretical characterization concerning the shadow developments.

Theorem (II.3): Let $\left\{u_{n}\right\}_{n \in \mathbf{N}}$ be an internal sequence of nonzero terms such that $\frac{u_{n+1}}{u_{n}} \cong 0$ for every $n \in N$, then $\sum_{n=0}^{\infty} u_{n}=u_{o}(1+\alpha) \quad(\alpha \cong 0)$

\section{Proof:}

Since $\sum_{n=0}^{\infty} u_{n}=u_{o} \sum_{n=0}^{\infty} \frac{u_{n}}{u_{o}}$. Set $v_{n}=\frac{u_{n}}{u_{o}}$ 
Then $v_{o}=1$ and $v_{n} \cong 0$ for every $n \in N$.Moreover for every $n \in N$ we have $\quad v_{n}=\frac{u_{n}}{u_{n-1}} \frac{u_{n-1}}{u_{n-2}} \ldots \frac{u_{1}}{u_{o}}<s$, where $s$ is a standard number greater than zero.

Since $\sum_{n=0}^{\infty} v_{n} \cong 1, \quad$ hence $\sum_{n=0}^{\infty} u_{n}=u_{o}(1+\alpha),(\alpha \cong 0)$

Theorem (II.4): Let $\left\{u_{n}\right\}_{n \in \mathbf{N}}$ be an internal sequence such that $\mathrm{u}_{\mathrm{o}}$ is limited, $u_{n} \cong 0$ for every $n \geq 1$, and such that the ratio of two nonzero successive terms is infinitesimally zero. Then $\sum_{n=0}^{\infty} u_{n} \cong u_{o}$

Proof: Let $p$ be the minimal index such that $u_{p} \neq 0$. By using Theorem (II.3), we get $\sum_{n=0}^{\infty} u_{n}=(1+\alpha) u_{p}$.If $\mathrm{p}=0$, then $(1+\alpha) u_{p}=u_{o}(1+\alpha) \cong u_{o}$.If $p>0$, since $u_{n} \cong 0$ for every $n \geq 1$ then $(1+\alpha) u_{p} \cong 0$, but $u_{o} \cong 0$, so $(1+\alpha) u_{p} \cong u_{o}$. Thus $\sum_{n=0}^{\infty} u_{n} \cong u_{o}$

\section{Remark (II.5):}

The statement of the Theorems (II.3) and (II.4) remains true if the sum is taken only up to certain index or if we mitigate the conditions on the ratio $\frac{u_{n+1}}{u_{n}}$. Thus we have, for example, the following theorem

Theorem (II.6): Let $\left\{u_{n}\right\}_{n \in \mathbf{N}}$ be an internal sequence and $k \in N$. Suppose that $u_{o}$ is limited, $\frac{u_{p}}{u_{o}} \cong 0$, when $p>0$ is the smallest index for which $u_{p} \neq 0$, and that the ratio of two nonzero successive terms of indexes included between $p$ and $k$ is $\quad<(\cong) 1$. Then $\sum_{n=0}^{k} u_{n} \cong u_{o}$. 


\section{REFERENCES}

[1] Copson, E. T. (1976), Asymptotic Expansions, England: Cambridge University press.

[2] Diener, M. \& Van Denberg, I. "Halos et galaxies une extension du lemma de Robinson", Compte Rendus de I'acadimie de Science de Paris, t.293 Serie, (1983) pp. 385-388.

[3] Hrbacek, K. (1979), "Nonstandard set theory", Amer. Math. Monthly, Vol.86, pp. 659-677.

[4] Ismail, T .H. "A characterization of a good approximation”, J. Ed. \& Sc., Vol.42, (2000).

[5] Lutz, R. \& Goze, M.; Nonstandard analysis, Practical Guide With Applications, Lecture Notes in Mathematics, No.881, SpringerVerlage, (1981).

[6] Nelson, E.; "Internal set theory: A new approach to nonstandard analysis", Bull. Amer. Math. Soc., Vol.83, No.6, (1977) pp.11651198.

[7] Robinson, A. (1974), Nonstandard Analysis $2^{\text {Ed. }}$, North - Holland, Amsterdam: Pub. Comp.

[8] Stroyan, K. D. \& Luxemburg, W. A. (1976), Introduction to the Theory of Infinitesimals", New York: Academic Press, 